\title{
Combining Low and High Mass Ion Accumulation for Enhancing Shotgun Proteome Analysis by Accurate Mass Measurement
}

\author{
Richard L. Wong and I. Jonathan Amster \\ Department of Chemistry, University of Georgia, Athens, Georgia, USA
}

\begin{abstract}
A simple procedure is described that increases sensitivity and dynamic range for the analysis of a proteome batch digest by FT-ICR mass spectrometry. Ions at the low and high mass ranges are preferentially collected using two different sets of tuning conditions. By combing data collected using tuning conditions that favor low mass $(m / z<2000)$ and high mass $(m / z>2000)$ ions, 277 proteins are identified for a whole cell lysate of Methanococcus maripaludis in a single HPLC-MALDI FT-ICR mass spectrometry experiment, a 70\% improvement compared with previous analyses using a wide mass range acquisition. This procedure improves the detection of low abundance ions and thereby increases the range of proteins that are observed. Because the observed mass range is effectively narrower for each spectrum, mass calibration is more accurate than for the standard method that provides a wide range of masses. The trap plate potential on the analyzer cell may be set to a higher value than used for wide mass range measurements, increasing the ion capacity of the analyzer cell and extending the dynamic range, while still maintaining mass accuracy. (J Am Soc Mass Spectrom 2006, 17, 205-212) (C) 2006 American Society for Mass Spectrometry
\end{abstract}

$\mathrm{P}$ roteomics is the study of the entire protein composition of a cell, tissue, or organism. A primary goal in proteomics is to monitor changes in protein expression in response to perturbations such as temporal evolution, environmental stress, or gene mutation. Considerable research effort is directed at accurately quantifying protein abundance changes in a high-throughput manner. The conventional proteomics methodology of combining two-dimensional polyacrylamide gel electrophoresis (2D-PAGE) separation with subsequent mass spectrometry is very labor-intensive and time-consuming, and has significant sensitivity and dynamic range limitations [1-5]. An approach that has generated considerable interest for higher throughput consists of a whole proteome enzymatic digestion followed by analysis that couples 1-D or 2-D liquid chromatography with mass spectrometry. Peptide identification is usually achieved by tandem mass spectrometry [4-6], or more recently, by accurate mass measurement using Fourier-transform ion cyclotron resonance (FT-ICR) mass spectrometry [2, 3, 7-13]. In the accurate mass measurement approach, proteins are identified by matching the measured peptide mass values with those predicted from an in silico digest of all proteins from

Published online January 18, 2006

Address reprint requests to Dr. I. J. Amster, Department of Chemistry, University of Georgia, Athens, GA 30602-2556, USA. E-mail: jamster@uga.edu the subject genome [2, 3, 7-10]. Since the identification relies only on mass values, the throughput of this approach is considerably higher because more than 100 peptide masses per mass spectrum can be measured and identified, while tandem mass spectrometry approach requires a one-by-one analysis of each peptide. In addition, the accurate mass measurement approach is less biased toward more abundant peptides compared with tandem mass spectrometry identification, in which the most abundant peptides are generally selected for analysis by the software that controls the online HPLC MS/MS measurement. The accurate mass measurement approach provides a high throughput alternative, and reliable quantification can be made with stable isotope labeling $[3,7,8,10]$. The peptide identification specificity is directly related to the mass accuracy level that serves as the primary search constraint. The normalized liquid chromatography elution time for a particular peptide can serve as an additional constraint for greater identification specificity [11-13]. Alternatively, the number of nitrogen in a peptide has also been suggested as a search constraint [7]. Identification specificity by incorporating the nitrogen stoichiometry improves by a factor of 2 to 3 times for an organism with $\sim 2000$ open reading frames (Parks, B. A.; Amster, I. J., manuscript in preparation).

Another challenge in proteomics is the ability to detect and monitor changes in the expression level of low abundance proteins. The detection limit for low 
abundance proteins is restricted by both sensitivity and dynamic range, which are coupled to one another. Therefore, the number of proteins that can be efficiently detected (observable sample size) is less than the actual number of proteins in the sample. Various detection improvements have been proposed for FT-ICR mass spectrometry. By temporarily reducing the trapping potential on an analyzer cell to zero, Hogan and Laude showed that signals for the low abundance ions in complex organic mixtures are amplified while the signals for the high abundance ions remain constant [14]. This increases the effective dynamic range for detection. McLafferty and coworkers demonstrated a 100-fold sensitivity improvement by remeasuring heavy ions $(m / z=2000)$ via collisional relaxation [15]. Amster and coworkers were the first to apply quadrupolar excitation and axialization in a FT-ICR cell for ion remeasurment [16, 17]. Signal-to-noise improved more than a factor of 10 by remeasuring the same ion packet 200 times. Marshall and coworkers increased sensitivity by applying quadrupolar excitation to the analyzer cell to relax the trapped ion motion [18], and to remeasure the same ion population to achieve attomole biomolecule detection [19]. Smith and coworkers expanded dynamic range by two orders of magnitude through accumulating low abundance ions in an analyzer cell via application of quadrupolar excitation in the form of bandlimited noise waveforms [20]. Applying a similar scheme to the external ion accumulation region, Smith and coworkers developed a technique called dynamic range enhancement applied to mass spectrometry (DREAMS), where a RF potential is applied to the external quadrupole to eject the most abundant species [10, 21-24]. A number of researchers have demonstrated signal-to-noise improvement via FT-ICR in-cell accumulation for MALDI generated ions [25-27]. Kelleher and coworkers demonstrated external ion accumulation with electrospray ionization using a quadrupole mass filter with a window of $m / z 40$ which provided a 1.5-fold improvement in signal-to-noise for the 11+ charge state of single protein bovine ubiquitin, but a 24-fold improvement for a complex Methanococcus jannaschii proteome sample compared to a broadband measurement [28]. The sensitivity improvement results from reserving the ion capacity of the analyzer cell for the ions of interest (within the mass filtering window), thus the improvement is greatest for complex mixtures. Zientek and Eyler used an external octopole ion guide with low RF frequency to discriminate against low mass, high abundance ions from a glow discharge source for elemental analysis [29].

Here, we describe a procedure which relies on mass discrimination from the ion flight time between an external hexapole and a FT-ICR analyzer cell to enhance the dynamic range for measuring peptides in a complex mixture. Ions in the low $(\mathrm{m} / \mathrm{z}=900-2500)$ and high $(\mathrm{m} / \mathrm{z}$ $=1500-4000)$ mass ranges are preferentially collected in the analyzer cell using two different sets of ion transfer and trapping parameters. This mass selective ion trapping method is used to analyze a proteome from Methanococcus maripaludis, and shown to improve ion detection and protein coverage.

\section{Experimental}

\section{Mass Spectrometry}

Mass spectra were collected on a 7 tesla BioApex Fourier-transform ion cyclotron resonance (FT-ICR) mass spectrometer (Bruker Daltonics, Billerica, MA) equipped with an intermediate pressure Scout100 MALDI source. Argon gas was pulsed into the source region during matrix-assisted laser desorption/ionization (MALDI) events to enhance ion accumulation in the hexapole region and to reduce the kinetic and internal energy of the ions. After accumulation, ions were released from the hexapole region by reducing the voltage applied to the hexapole exit electrode, then directed to the analyzer cell by using electrostatic ion optics, and trapped in the analyzer cell using Sidekick [30]. Mass spectra were collected by accumulating ions from 12 MALDI laser shots per scan and 12 scans were co-added for each recorded spectrum. Spectra were collected using high mass and low mass enhancing conditions (vide infra) on alternate acquisitions for each sample spot.

\section{High Performance Liquid Chromatography (HPLC)}

Reversed-phase liquid chromatography separations of peptide mixtures were carried out on an UltiMate Plus system (Dionex LC Packings, Sunnyvale, CA), using a $75 \mu \mathrm{m}$ i.d. $\times 10 \mathrm{~cm} \mathrm{C18}$ nanocolumn with $3 \mu \mathrm{m}$ particles and $300 \AA$ pore size (Dionex LC Packings, Sunnyvale, CA). Mobile phase A was $95 / 5 / 0.1 \%$ water/acetonitrile/trifluoroacetic acid (by volume), and mobile phase B was $100 \%$ acetonitrile. The proteome sample was concentrated in a C18 precolumn, injected onto the C18 nanocolumn, and separated using a 100 min gradient at a flow rate of $300 \mathrm{~nL} / \mathrm{min}$. Mobile phase B started at $0 \%$ in the beginning of the experiment and was ramped to $5 \%$ at $10 \mathrm{~min}, 15 \%$ at $40 \mathrm{~min}, 35 \%$ at $60 \mathrm{~min}, 75 \%$ at 90 $\mathrm{min}$, and $100 \%$ at $100 \mathrm{~min}$. Eluate was applied to sequential positions on a MALDI stainless steel target with a spotting robot (Probot, Dionex LC Packings, Sunnyvale, CA) at a $30 \mathrm{~s}$ interval for the first 86 fractions and at every $60 \mathrm{~s}$ for fractions $87-111$.

\section{Materials and Sample Preparation}

2,5-dihydroxybenzoic acid (DHB) and dithiothreitol (DTT) were purchased from Lancaster (Pelham, NH) and Sigma (St. Louis, MO), respectively. Trypsin was purchased from Promega (Madison, WI). Chicken egg white albumin (ovalbumin) was purchased from Sigma. The analyzed proteome was a whole cell lysate from Methanococcus maripaludis, which was grown on minimal media with ammonium sulfate as the sole source of 
nitrogen. Wild type M. maripaludis was grown to midlogarithmic and stationary stages in ammonium sulfate with naturally occurring isotopic composition and with $98 \%{ }^{15} \mathrm{~N}$-enriched composition, respectively. The cells were concentrated by centrifugation at $10,000 \times g$ for 30 min and the compacted cells were lysed using a French pressure cell. DNA was digested and removed from the extract by adding DNAase (10 $\mu \mathrm{M}$ final concentration) to the sample followed by centrifugation. Protein concentrations were determined via photometrically measuring at $562 \mathrm{~nm}$ using a bicinchoninic acid protein assay kit (Pierce, Rockford, IL) [31]. Equal amounts of protein extracts from the two growth stages were mixed together before batch trypsinolysis.

Small molecules were removed from protein samples using gel-filtration with Sephadex G-25 (Aldrich, St. Louis, MO) in a $3 \mathrm{~mL}$ spin column. The samples were prepared at $\sim 1 \mathrm{mg} / \mathrm{mL}$ concentration and heat denatured at $90{ }^{\circ} \mathrm{C}$ for $5-10 \mathrm{~min}$. Disulfide bonds were reduced using $5 \mathrm{mM}$ DTT at $70^{\circ} \mathrm{C}$ for $1 \mathrm{~h}$. Denatured samples were digested overnight at $37^{\circ} \mathrm{C}$ using trypsin at a 1:50 protease:protein ratio (by mass). $400 \mathrm{~nL}$ of the digested ovalbumin solution was spotted onto stainless steel MALDI plates, for use as a mass calibrant. The digested proteome sample was separated using reversed-phase HPLC before spotting. Samples were allowed to dry before adding $400 \mathrm{~nL}$ of MALDI matrix solution to each spot. The matrix solution was prepared using $10 \mathrm{mg}$ of DHB dissolved in $60 \mu \mathrm{L}$ of 50:50:0.1\% water:acetonitrile:trifluoroacetic acid solution (by volume).

\section{Protein Identification for Methanococcus maripaludis}

The accurate masses of the tryptic peptides were measured using MALDI FT-ICR mass spectrometry. The number of nitrogen atoms in each peptide was determined from the mass separation between the monoisotopic peaks of the peptide and its ${ }^{15} \mathrm{~N}$-enriched counterpart. The identification of the peptides was automated using software that was written in-house. The routines for data analysis are written in Java, and are available upon request. Predicted tryptic fragments were calculated for the 1722 possible proteins of $M$. maripaludis, allowing up to one possible missed-cleavage or two missed-cleavages if two basic residues (lysine or arginine) are next to each other. A measured mass peak is considered to be identified when only one predicted tryptic peptide has both a mass within 10 ppm of the measured value and has the observed nitrogen stoichiometry. The $10 \mathrm{ppm}$ mass confidence window is chosen because the standard deviation for using external calibration on our instrument is $\sim 3.5$ $\mathrm{ppm}$. Therefore, a $10 \mathrm{ppm}$ confidence window is three times the standard deviation, corresponding to a $99 \%$ confidence limit for the measured mass. The details of this method will be described elsewhere (Parks, B. A.; Amster, I. J., manuscript in preparation).

\section{Procedures for Mass Selective Enhancement}

The mass spectrometer is tuned to preferentially detect the low and the high mass ions for each MALDI spot. Lighter and heavier ions are preferentially collected in the analyzer cell using slightly different RF voltages on the source hexapole, $200 \mathrm{~V}$ versus $250 \mathrm{~V}$, and by using different ion extraction times, $3600 \mu \mathrm{s}$ versus $4600 \mu \mathrm{s}$, respectively. The ion extraction time is the period between the reduction of the trapping potential applied to the hexapole exit lens and the termination of the Sidekick potential at the analyzer cell entrance. The ion extraction time is significantly longer than the actual ion flight time between the hexapole region and the analyzer cell. Ions do not leave the hexapole region as a tight packet; instead, they diffuse slowly via ion-ion repulsion, producing a lengthy exit time profile [32]. As the ions drift out of the hexapole region, they are separated by their mass-dependent velocities during the transfer to the analyzer cell, as shown in Figure 1. The convolution of their exit time distribution with their flight time profile to the analyzer reduces the mass discrimination that might otherwise occur by sampling different extraction times. Nevertheless, a mass discrimination is observed. In addition, a $0.60 \mathrm{~V}$ trap plate potential is used for trapping low mass ions and a 0.32 $\mathrm{V}$ for high mass ions. A higher trap plate potential increases the ion capacity of the analyzer cell [28, 33-35], allowing more ions to be detected. On the other hand, some non-ideal effects in FT-ICR, such as radial ion lost [34, 35], space-charge frequency shift [36-38], and ion coalescence $[39,40]$ become more apparent when using a higher trapping potential, making mass calibration less accurate, especially at higher mass-tocharge. Because the mass collection in these experiments here is enhanced for the low and high mass ranges, a lower trapping potential is used for the high mass enhancing conditions to ensure high mass measurement confidence for the high mass ions, while a higher trapping potential is utilized for the low mass enhancing conditions to increase the number of ions that can be trapped, and thereby increasing the dynamic range for low mass ions. The capability to analyze more ions is important at low masses because significantly more tryptic peptides are produced in the lower mass range, and because the lower mass peptide ions have lower identification specificity (that is, a smaller fraction of peptides can be identified by accurate mass measurement at lower masses).

\section{Results and Discussion}

For these experiments, the mass bias effect is pronounced for MALDI samples which have strong signals across a wide mass range, as seen in Figure 2. The upper panel shows a HPLC fraction of the tryptic digest of $M$. 


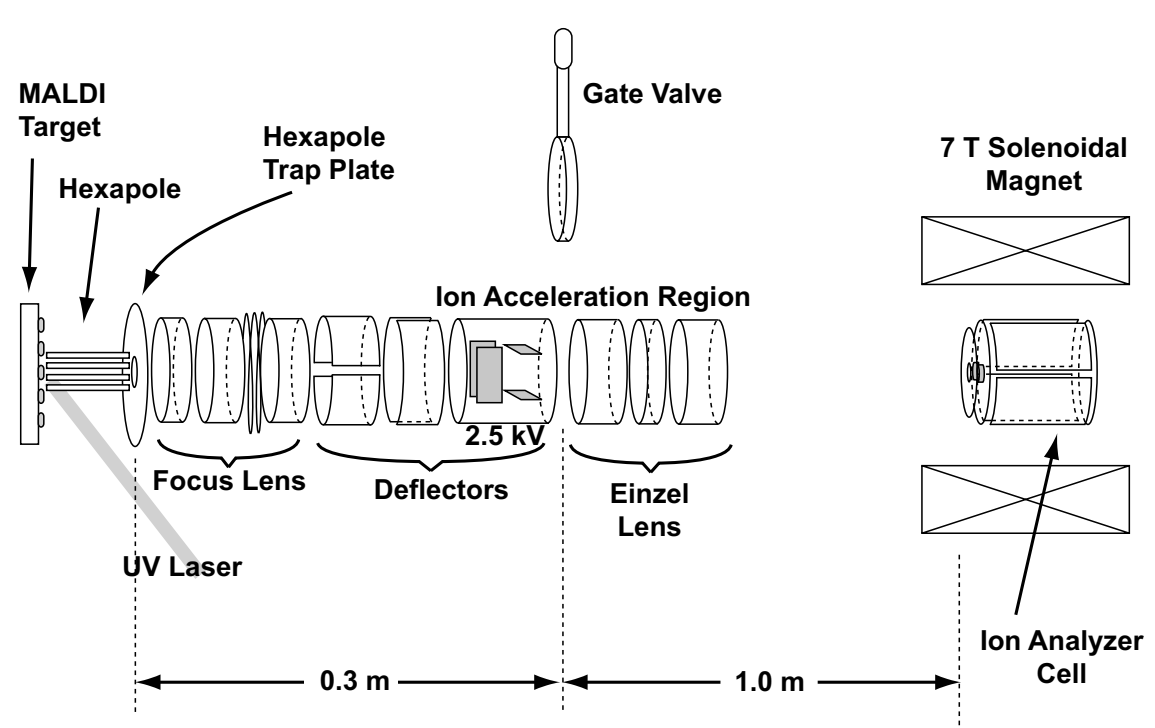

Figure 1. A schematic showing the regions where ions are generated, transferred, and detected.

maripaludis acquired with the low mass enhancing conditions (ion extraction time $=3600 \mu \mathrm{s}$, hexapole RF voltage $=200 \mathrm{~V}$, trap plate potential $=0.60 \mathrm{~V}$ ), while the lower panel shows the same sample acquired with the high mass enhancing conditions (ion extraction time $=4600 \mu \mathrm{s}$, hexapole $\mathrm{RF}$ voltage $=250 \mathrm{~V}$, trap plate potential $=0.32 \mathrm{~V}$ ). A group of ions above $\mathrm{m} / \mathrm{z} 2500$ is more efficiently detected by using the high mass enhancing conditions. For MALDI samples which produce intense peaks in only the middle of the mass range, the mass enhancing effect is less apparent unless the region of interest is expanded, as demonstrated in Figure 3a and $\mathrm{b}$. However, peaks between $\mathrm{m} / \mathrm{z} 3260$ to 3305 , which are undetectable using the low mass enhancing conditions, are easily identified using the high mass enhancing conditions as seen in Figure $3 c$ and $d$. Mass calibration is significantly affected by the change in trapping potential, and so two different calibration functions are used. Although the mass bias produced by the two tuning conditions is apparent, this procedure does not produce a sharp cut-off in the detected mass range for the reasons explained above. As seen in Figure 2, low mass ions that show up with high intensity under the low mass enhancing conditions appear as weak intensity peaks under the high mass enhancing conditions, and vice versa. However, less abundant ions at the two ends of the mass range are expected to only appear in the corresponding enhanced mass spectra, as seen in Figure 3.

\section{Categorization of Low Mass and High Mass Enhancements}

Using the combined low and high mass enhancing acquisition on a batch digest of a whole cell lysate of $M$. maripaludis, 3842 peak pairs are detected from 111 HPLC fractions. This sample utilizes ${ }^{15} \mathrm{~N}$ metabolic labeling, and so each peptide appears as a pair of peaks.
The number of peak pairs that can be assigned to one or more predicted peptides is 3111, and 1362 of these can be each assigned to a single peptide. However, many peak pairs are redundant, as they appear in multiple MALDI spots. Moreover, some peptides appear in both the low and high mass enhanced spectra. Consequently, the numbers of identified peptides and identified proteins are 394 and 277, respectively. This is a vast improvement compared with our previous measurements, where the numbers of identified peptides and proteins in a single reverse-phase HPLC/MALDI-MS analysis of the whole cell lysate are $\sim 220$ and $\sim 160$, respectively (Parks, B. A.; Amster, I. J., manuscript in preparation). The number of identified peptides is usually greater than that of the identified proteins because some proteins are identified by more than one peptide. The ratio between the numbers of identified proteins and peptides is maintained at $\sim 71 \%$ for data collected using the previous and the mass selective enhancing conditions. The significance of this ratio will be discussed below.

To better understand the impact of using the two sets of tuning conditions to acquire data, we have separately analyzed the low mass and high mass enhanced data. 231 proteins are identified using peak pairs found with the low mass enhancing conditions, whereas 210 proteins are identified using data collected with the high mass conditions. Approximately $60 \%$ of the total identified proteins are found in both sets of peak lists, while $40 \%$ of the identified proteins are from peptides found in only one of the two peak lists. The strong protein identification overlap between the two peak lists is largely due to ions in the middle mass range which are detected efficiently by either set of the tuning conditions.

It should be noted that the number of proteins identified from either set of the enhancing conditions is noticeably higher than the usual 160 proteins identified 


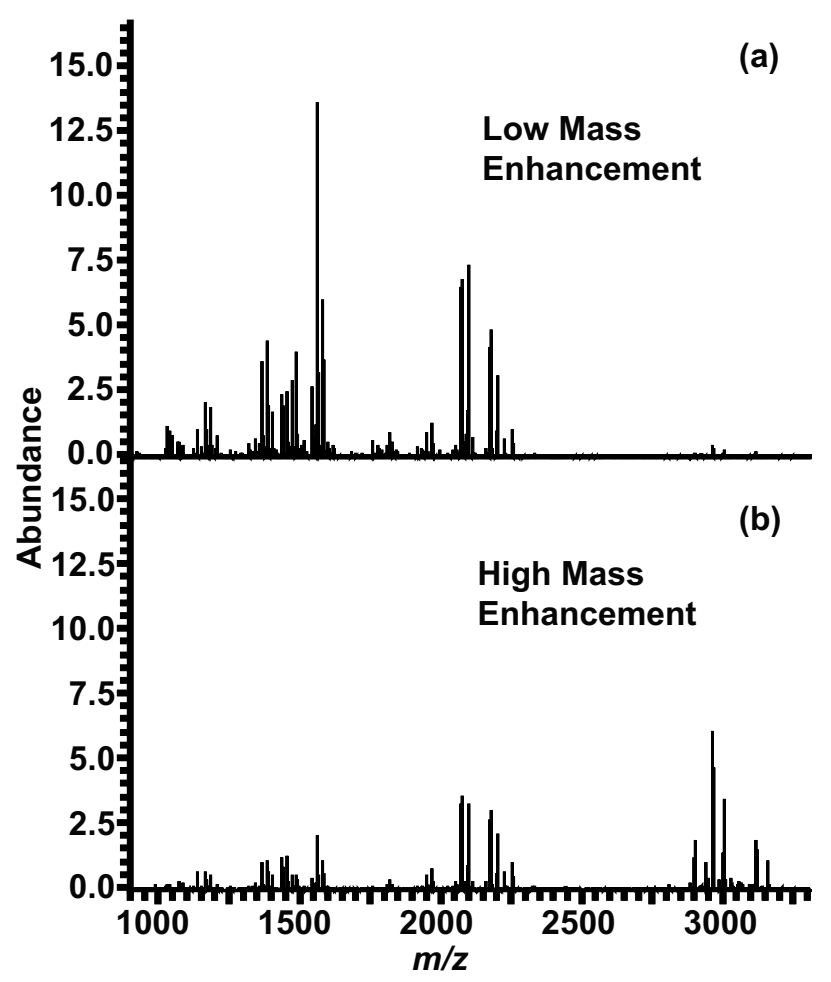

Figure 2. Mass spectra at HPLC elution time 53.5 min collected using (a) low mass enhancing conditions, and (b) high mass enhancing conditions. Spectra are signal-averaged 12 times.

from previous wide mass range detection, which suggests that the narrower mass range acquisition provides better sensitivity for low abundance ions. The mass distributions of peak pairs found using the low and high mass enhancing conditions are shown in Figure 4a. The low mass enhancing conditions have a significant sensitivity advantage for ions of $m / z<1600$, and the high mass enhancing conditions have an advantage for $m / z>2600$. In addition, spectra collected using the low mass enhancing conditions have greater overall signals, which, in part, is because there are more tryptic fragments at lower mass, and partly because the trap plate potential is higher than that used for the high mass enhancing conditions $(0.60 \mathrm{~V}$ versus $0.32 \mathrm{~V})$, which increases the ion capacity of the analyzer cell. The mass distributions of peptides identified from data collected using the two enhancing conditions are shown in Figure $4 \mathrm{~b}$.

\section{Benefits of the Additional Mass Peaks}

To better understand the manner in which this approach increases protein identification, it is useful to categorize the measured mass values as exclusive peak pairs, those which are found only using one of the enhancing conditions versus mutual peak pairs, those found in both sets of data. The number of proteins identified based on searching with the mutual peak pairs list is 187, while 124 proteins are found using only the exclusive peak pairs list. Since the total number of identified proteins remains 277 , fewer identified proteins are shared between the mutual and exclusive peak lists than between the low and high mass peak lists. Only $12 \%$ of the total identified proteins are overlapped between the mutual and exclusive peak pair results, which demonstrates that proteins are more effectively categorized. In short, a majority of the additional peptide peaks detected in only the low or the high mass enhancing condition (exclusive) belong to a different group of proteins than those found using peaks that are found in both sets of conditions (mutual).

The benefit of collecting data using the two experimental conditions can be seen by categorizing peak pairs which are found mutually in the low and high mass enhanced spectra versus those that are found exclusively using one of the tuning conditions, Figure 5a. As expected, exclusive peak pairs outnumber the mutual peak pairs at the extremes of mass-to-charge. Although the exclusive peak pairs list is only one-third the size of the mutual peak pairs list, the total number of peptides identified by the exclusive peak pairs is one-half that of the mutual peak pairs, as shown in Figure $5 \mathrm{~b}$. Hence, the mutual peak pairs must have a higher redundancy rate than the exclusive list does (by definition, mutual peak pairs are those found using both enhancing conditions. To avoid double-counting these peptides, only the peak pairs from the high mass enhanced list are recorded for the mutual peak list. The redundancy rate in Figure 5 only represents that between different HPLC elution times). The reason for the higher redundancy rate for mutual peak pairs is that mutual peak pairs have a larger representation of high abundance peptide ions, whereas the exclusive peak pairs have a much higher representation of the low abundance peptide ions. Because of their stronger ion signals, the mutual peak pairs have a greater tendency to appear in multiple MALDI spots, producing redundant peak pairs. Although mass spectrometric abundance does not strictly translate to solution-phase population because the ionization efficiency is different for each peptide, a correlation does exist between the gas-phase and solution-phase abundances over such a large sample size. These data suggest exclusive peak pairs, in general, are more representative of peptides from lower abundance proteins compared with those from mutual peak pairs. For example, Asp-tRNAAsn/ Glu-tRNAGln amidotransferase subunit B (MMP0946 in the M. maripaludis sequence database) is found via identification of two peptides at $\mathrm{m} / \mathrm{z}=1581.7338$ and 2756.3686, which are exclusively detected using the low and high mass enhancing conditions, respectively. No peptide from the mutual peak pairs list was identified for this protein. On the other hand, S-layer protein (MMP0383), a known high abundance protein, is identified via five peptide ions, four of which are found mutually in the low and high mass enhancing spectra, and only one is exclusively found.

The improvement of the observable protein sample size is also demonstrated by the ratio between the 


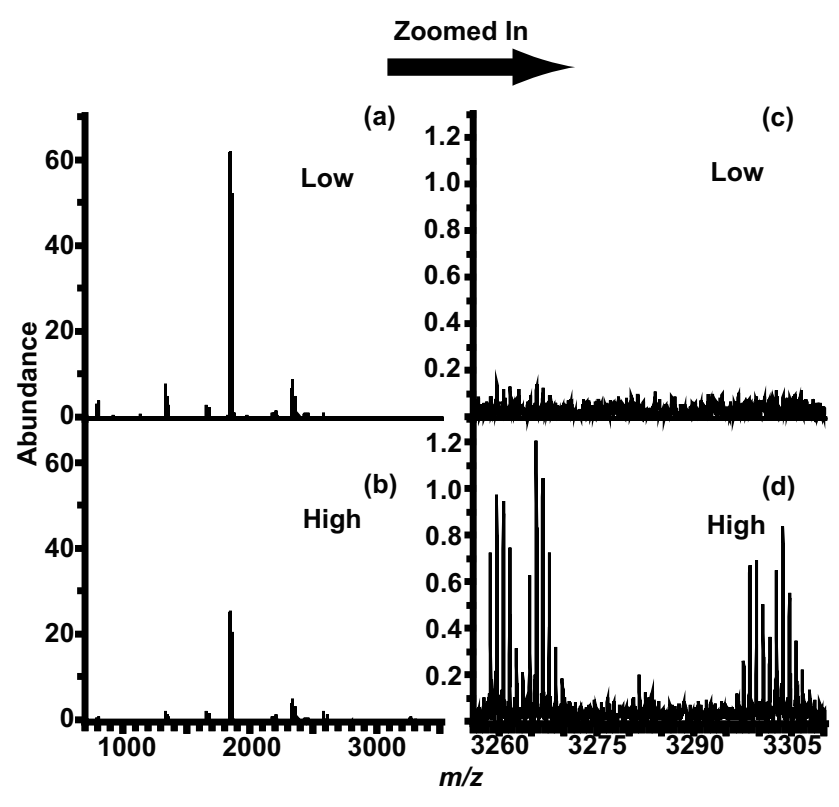

Figure 3. Mass spectra collected at HPLC elution time $70.5 \mathrm{~min}$ using (a) low mass enhancing conditions, (b) high mass enhancing conditions, and (c), (d) their corresponding spectra expanded at $\mathrm{m} / \mathrm{z}$ 3255-3310. Spectra are signal-averaged 12 times.

numbers of identified proteins and identified peptides. Although almost twice as many peptides are identified using the combined low and high mass enhancing procedure compared with the wide mass scan mode (394 versus 220), the ratio of identified proteins versus identified peptides is unchanged. If the observable protein sample size is unchanged, this ratio must de-

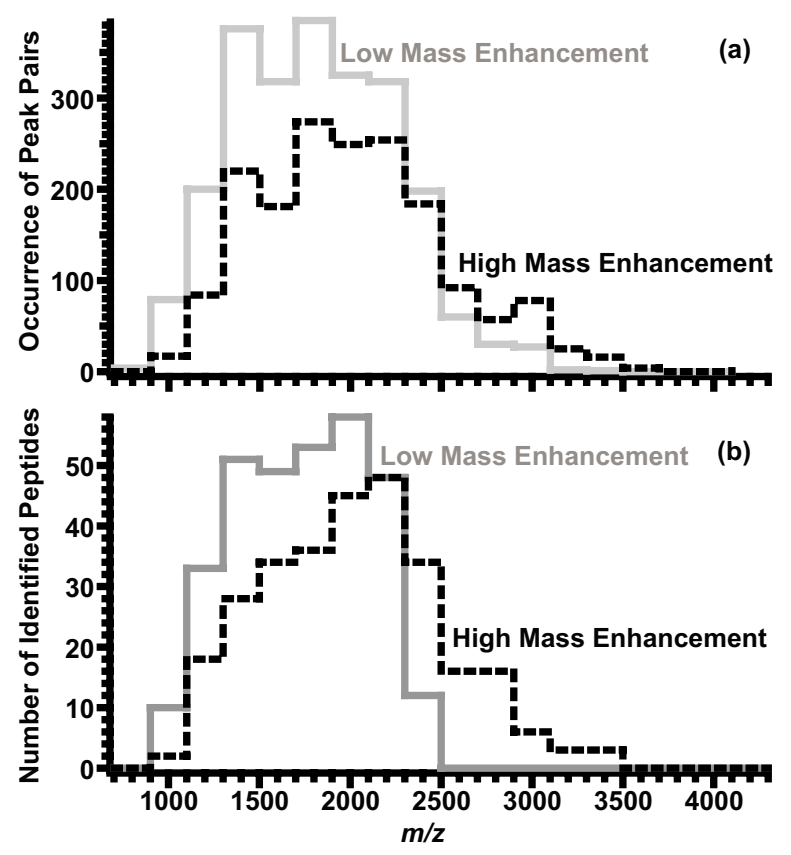

Figure 4. Histograms for (a) peak pair occurrence, (b) number of identified peptides, versus measured mass for spectra collected using low mass enhancing conditions (solid line), and high mass enhancing conditions (dashed line).

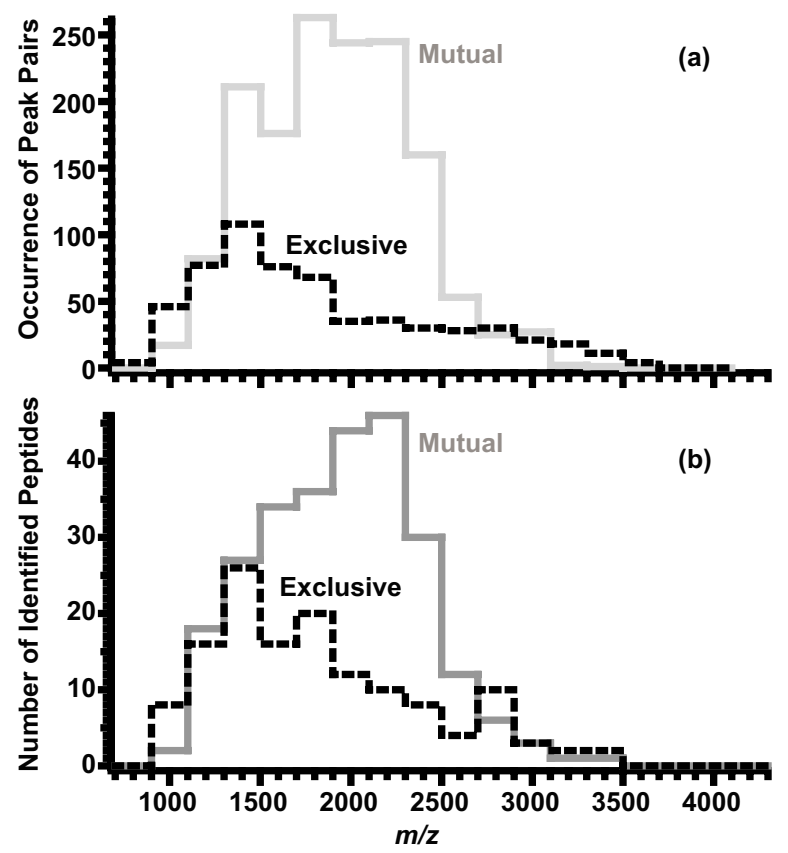

Figure 5. Histograms for (a) peak pair occurrence, (b) number of identified peptides, versus measured mass for the peak pairs found mutually (solid line) in both low and high mass enhanced spectra, and those found exclusively (dashed line) in one of the enhanced spectra.

crease as the number of identified peptides increases. The fact that the ratio is unchanged here strongly suggests that the observable protein sample size increases when the mass selective enhancing method is applied by increasing the sensitivity for the lower abundance proteins. Therefore, more proteins can be identified from a narrower mass spectrum with higher sensitivity than by using a full range low sensitivity mass scan.

It is not unusual to increase protein identification by acquiring data from the same sample multiple times in multidimensional protein identification technology (MudPIT) experiments [41-43]. In our case, due to the smaller populations of the low abundance ions the relative signal fluctuation for these species is substantial for each measurement, which means the relative ion signal observed for these species can be much larger or smaller than the relative abundance in the sample. Thus, performing multiple measurements at even the same conditions is expected to increase the observable sample size. However, we believe that the observed increase in the number of identified proteins is from the expansion of the detection dynamic range at the low and high ends of mass-to-charge. The relative protein identification improvement from the exclusive peaks is demonstrated by examining the ratio between the number of peptides identified from the combined peak pairs and that from the mutual peak pairs as a function of the measured mass, as shown in Figure 6a. The high ratio observed at the extremes of the mass range shows that the exclusive peak pairs contribute the most to protein 
identification at the two ends of the mass-to-charge range. By partitioning the identified peptides from the exclusive peak pairs into those collected using the low and high mass enhancing conditions, as shown in Figure $6 \mathrm{~b}$, one can see that the "additional" peptides collected using the two mass enhancing conditions strongly depend on the peptide mass. If the increase in protein identification is solely due to random sampling from two independent measurements, then the two peptide identification distributions in Figure $6 \mathrm{~b}$ would resemble the distributions in Figure 4. Instead, the additional low mass peptides are mostly collected using the low mass enhancing conditions, and vice versa for high mass peptides. This indicates the increase in protein identification benefit from the higher detection sensitivity at the two ends of the mass spectra.

\section{Conclusions}

Protein identification of a whole cell lysate is shown to improve by using a procedure that can be implemented with most FT-ICR mass spectrometers. 277 proteins are identified by combing data collected at the low and high mass enhancing conditions, which is a significant improvement compared with the 160 proteins identified without using this method. The improvement for protein identification is attributable to the ability to accumulate a narrower distribution of ions in the analyzer cell, therefore allowing more ions at a particular mass range to be detected. This effectively improves both the sensitivity and the dynamic range of FT-ICR mass spectrometry. This mass selective enhancing technique has certain advantages over the DREAMS technique. In contrast to DREAMS, small peaks that are near the most abundant peaks are not ejected, and ions do not undergo translational excitation and therefore avoid the possibility of fragmentation [44]. Under usual situations, mass accuracy deteriorates as trap plate voltage increases. In this case, because the mass range of interest is made narrower, the trap plate potential can be increased to expand the capacity of the analyzer cell without sacrificing mass accuracy.

Although ion time-of-flight effect is used to select the mass range in this work, quadrupole mass filtering [44-46] or stored waveform inverse Fourier transform (SWIFT) isolation [47] should be able to achieve similar results. Gated trapping method may substitute Sidekick trapping for better resolution of the time-of-flight separation of ions as they are injected into the analyzer cell. The mass selective enhancement may be applied to more than two mass ranges for higher sensitivity improvement. This can be achieved using an external quadrupole mass filter, as demonstrated by Kelleher and coworkers [28]. On the other hand, the time needed for spectra collection, the amount of sample consumption, and the amount of data to be processed increases with the number of mass windows. The advantage of this technique is not limited to proteomic studies. Generally speaking, this approach should benefit any

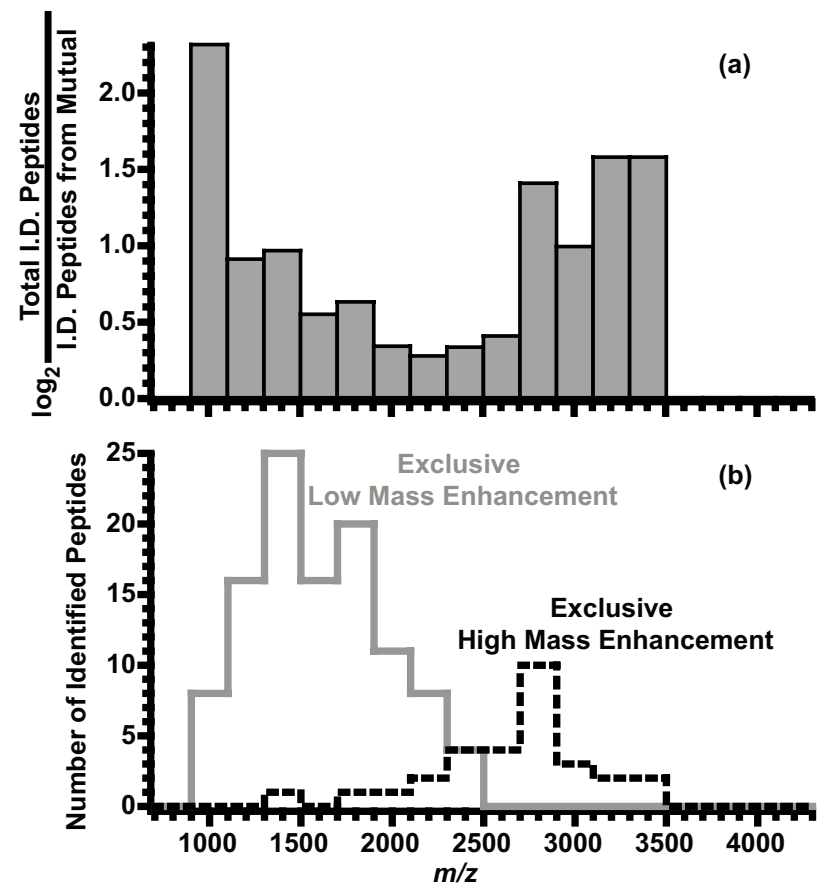

Figure 6. Histograms for (a) the ratio between numbers of identified peptides from entire peak pairs and from the mutual peak pairs alone, and (b) the numbers of peptides identified by exclusive peak pairs that are collected using the low (solid line) and high (dashed line) mass enhancing conditions.

experiment requiring higher sensitivity or greater dynamic range for complex mixture analysis.

\section{Acknowledgments}

The authors thank Professor W. B. Whitman and Dr. I. Porat (Department of Microbiology, University of Georgia) for providing proteome samples of $M$. maripaludis. The authors are also grateful for financial support through the National Science Foundation (grant CHE-0316002) and National Institutes of Health (grant R01-RR 019767).

\section{References}

1. Gygi, S. P.; Aebersold, R. Mass Spectrometry and Proteomics. Curr. Opin. Chem. Biol. 2000, 4, 489-494.

2. Conrads, T. P.; Anderson, G. A.; Veenstra, T. D.; Pasa-Tolic, L.; Smith, R. D. Utility of Accurate Mass Tags for Proteome-Wide Protein Identification. Anal. Chem. 2000, 72, 3349-3354.

3. Smith, R. D.; Anderson, G. A.; Lipton, M. S.; Pasa-Tolic, L.; Shen, Y. F.; Conrads, T. P.; Veenstra, T. D.; Udseth, H. R. An Accurate Mass Tag Strategy for Quantitative and High-Throughput Proteome Measurements. Proteomics 2002, 2, 513-523.

4. Yates, J. R. Mass Spectrometry-from Genomics to Proteomics. Trends Genet. 2000, 16, 5-8.

5. McDonald, W. H.; Yates, J. R. Shotgun Proteomics and Biomarker Discovery. Disease Markers 2002, 18, 99-105.

6. Washburn, M. P.; Ulaszek, R.; Deciu, C.; Schieltz, D. M.; Yates, J. R. Analysis of Quantitative Proteomic Data Generated via Multidimensional Protein Identification Technology. Anal. Chem. 2002, 74, 16501657.

7. Gao, H. Y.; Shen, Y. F.; Veenstra, T. D.; Harkewicz, R.; Anderson, G. A.; Bruce, J. E.; Pasa-Tolic, L.; Smith, R. D. Two-Dimensional Electrophoretic/Chromatographic Separations Combined with Electrospray Ionization FTICR Mass Spectrometry for High Throughput Proteome Analysis. J. Microcolumn Sep. 2000, 12, 383-390.

8. Conrads, T. P.; Alving, K.; Veenstra, T. D.; Belov, M. E.; Anderson, G. A.; Anderson, D. J.; Lipton, M. S.; Pasa-Tolic, L.; Udseth, H. R.; Chrisler, W. B.; Thrall, B. D.; Smith, R. D. Quantitative Analysis of Bacterial and 
Mammalian Proteomes Using a Combination of Cysteine Affinity Tags and N-15-Metabolic Labeling. Anal. Chem. 2001, 73, 2132-2139.

9. Lipton, M. S.; Pasa-Tolic, L.; Anderson, G. A.; Anderson, D. J.; Auberry, D. L.; Battista, J. R.; Daly, M. J.; Fredrickson, J.; Hixson, K. K.; Kostandarithes, H.; Masselon, C.; Markillie, L. M.; Moore, R. J.; Romine, M. F.; Shen, Y. F.; Stritmatter, E.; Tolic, N.; Udseth, H. R.; Venkateswaran, A.; Wong, K. K.; Zhao, R.; Smith, R. D. Global Analysis of the Deinococcus radiodurans Proteome by Using Accurate Mass Tags. Proc. Natl. Acad. Sci. U.S.A. 2002, 99, 11049-11054.

10. Pasa-Tolic, L.; Harkewicz, R.; Anderson, G. A.; Tolic, N.; Shen, Y. F.; Zhao, R.; Thrall, B.; Masselon, C.; Smith, R. D. Increased Proteome Coverage for Quantitative Peptide Abundance Measurements Based upon High Performance Separations and DREAMS FTICR Mass Spectrometry. J. Am. Soc. Mass Spectrom. 2002, 13, 954-963.

11. Petritis, K.; Kangas, L. J.; Ferguson, P. L.; Anderson, G. A.; Pasa-Tolic, L.; Lipton, M. S.; Auberry, K. J.; Strittmatter, E. F.; Shen, Y. F.; Zhao, R.; Smith, R. D. Use of Artificial Neural Networks for the Accurate Prediction of Peptide Liquid Chromatography Elution Times in Proteome Analyses. Anal. Chem. 2003, 75, 1039-1048.

12. Strittmatter, E. F.; Ferguson, P. L.; Tang, K. Q.; Smith, R. D. Proteome Analyses Using Accurate Mass and Elution Time Peptide Tags with Capillary LC Time-of-Flight Mass Spectrometry. J. Am. Soc. Mass Spectrom. 2003, 14, 980-991.

13. Jacobs, J. M.; Mottaz, H. M.; Yu, L. R.; Anderson, D. J.; Moore, R. J.; Chen, W. N. U.; Auberry, K. J.; Strittmatter, E. F.; Monroe, M. E.; Thrall, B. D.; Camp, D. G.; Smith, R. D. Multidimensional Proteome Analysis of Human Mammary Epithelial Cells. J. Proteome Res. 2004, 3, 68-75.

14. Hogan, J. D.; Laude, D. A. Suspended Trapping Gas Chromatography/ Fourier Transform Mass Spectrometry for Analysis of Complex Organic Mixtures. J. Am. Soc. Mass Spectrom. 1990, 1, 431-439.

15. Williams, E. R.; Henry, K. D.; McLafferty, F. W. Multiple Remeasurement of Ions in Fourier-Transform Mass-Spectrometry. J. Am. Chem. Soc. 1990, 112, 6157-6162

16. Speir, J. P.; Gorman, G. S.; Pitsenberger, C. C.; Turner, C. A.; Wang, P. P.; Amster, I. J. Remeasurement of Ions Using Quadrupolar Excitation Fourier Transform Ion Cyclotron Resonance Spectrometry. Anal. Chem. 1993, 65, 1746-1752.

17. Pitsenberger, C. C.; Easterling, M. L.; Amster, I. J. Efficient Ion Remeasurement Using Broadband Quadrupolar Excitation FTICR Mass Spectrometry. Anal. Chem. 1996, 68, 3732-3739.

18. Guan, S. H.; Wahl, M. C.; Wood, T. D.; Marshall, A. G. Enhanced Mass Resolving Power, Sensitivity, and Selectivity in Laser Desorption Fourier-Transform Ion Cyclotron Resonance Mass Spectrometry by Ion Axialization and Cooling. Anal. Chem. 1993, 65, 1753-1757.

19. Solouki, T.; Marto, J. A.; White, F. M.; Guan, S. H.; Marshall, A. G. Attomole Biomolecule Mass Analysis by Matrix-Assisted Laser Desorption/Ionization Fourier Transform Ion Cyclotron Resonance. Anal. Chem. 1995, 67, 4139-4144.

20. Bruce, J. E.; Anderson, G. A.; Smith, R. D. "Colored" Noise Waveforms and Quadrupole Excitation for the Dynamic Range Expansion of Fourier Transform Ion Cyclotron Resonance Mass Spectrometry. Anal. Chem. 1996, 68, 534-541.

21. Belov, M. E.; Nikolaev, E. N.; Anderson, G. A.; Auberry, K. J.; Harkewicz, R.; Smith, R. D. Electrospray Ionization-Fourier Transform Ion Cyclotron Mass Spectrometry Using Ion Preselection and External Accumulation for Ultrahigh Sensitivity. J. Am. Soc. Mass Spectrom. 2001, $12,38-48$.

22. Belov, M. E.; Anderson, G. A.; Angell, N. H.; Shen, Y. F.; Tolic, N.; Udseth, H. R.; Smith, R. D. Dynamic Range Expansion Applied to Mass Spectrometry Based on Data-Dependent Selective Ion Ejection in Capillary Liquid Chromatography Fourier Transform Ion Cyclotron Resonance for Enhanced Proteome Characterization. Anal. Chem. 2001, 73, 5052-5060.

23. Harkewicz, R.; Belov, M. E.; Anderson, G. A.; Pasa-Tolic, L.; Masselon, C. D.; Prior, D. C.; Udseth, H. R.; Smith, R. D. ESI-FTICR Mass Spectrometry Employing Data-Dependent External Ion Selection and Accumulation. J. Am. Soc. Mass Spectrom. 2002, 13, 144-154.

24. Belov, M. E.; Anderson, G. A.; Wingerd, M. A.; Udseth, H. R.; Tang, K. Q.; Prior, D. C.; Swanson, K. R.; Buschbach, M. A.; Strittmatter, E. F.; Moore, R. J.; Smith, R. D. An Automated High Performance Capillary Liquid Chromatography-Fourier Transform Ion Cyclotron Resonance Mass Spectrometer for High-Throughput Proteomics. J. Am. Soc. Mass Spectrom. 2004, 15, 212-232

25. Mize, T. H.; Amster, I. J. Broad-Band Ion Accumulation with an Internal Source MALDI-FTICR-MS. Anal. Chem. 2000, 72, 5886-5891.
26. O'Connor, P. B.; Costello, C. E. Application of Multishot Acquisition in Fourier Transform Mass Spectrometry. Anal. Chem. 2000, 72, 5125-5130.

27. Kutz, K. K.; Schmidt, J. J.; Li, L. J. In Situ Tissue Analysis of Neuropeptides by MALDI FTMS In-Cell Accumulation. Anal. Chem. 2004, 76, 5630-5640.

28. Patrie, S. M.; Charlebois, J. P.; Whipple, D.; Kelleher, N. L.; Hendrickson, C. L.; Quinn, J. P.; Marshall, A. G.; Mukhopadhyay, B. Construction of a Hybrid Quadrupole/Fourier Transform Ion Cyclotron Resonance Mass Spectrometer for Versatile MS/MS above $10 \mathrm{kDa}$. J. Am. Soc. Mass Spectrom. 2004, 15, 1099-1108.

29. Zientek, K. D.; Eyler, J. R. Improved Sensitivity for Low Abundance Ions in Glow Discharge Fourier Transform Ion Cyclotron Resonance Mass Spectrometry Following Ion Preselection in an External Octopole Ion Guide. J. Anal. At. Spectrom. 2004, 19, 1513-1516.

30. Caravatti,, P. Method and Apparatus for The Accumulation of Ions in a Trap of an Ion Cyclotron Resonance Spectrometer, by Transferring the Kinetic Energy of the Motion Parallel to the Magnetic Field into Directions Perpendicular to the Magnetic Field; U.S. Patent. 4, 924089, 1990.

31. Smith, P. K.; Krohn, R. I.; Hermanson, G. T.; Mallia, A. K.; Gartner, F. H.; Provenzano, M. D.; Fujimoto, E. K.; Goeke, N. M.; Olson, B. J.; Klenk, D. C. Measurement of Protein Using Bicinchoninic Acid. Anal. Biochem. 1985, 150, 76-85.

32. Wilcox, B. E.; Hendrickson, C. L.; Marshall, A. G. Improved Ion Extraction from a Linear Octopole Ion Trap: SIMION Analysis and Experimental Demonstration. J. Am. Soc. Mass Spectrom. 2002, 13, 1304-1312.

33. Wood, T. D.; Schweikhard, L.; Marshall, A. G. Mass-to-Charge Ratio Upper Limits for Matrix-Assisted Laser Desorption Fourier-Transform Ion-Cyclotron Resonance Mass-Spectrometry. Anal. Chem. 1992, 64, 1461-1469.

34. May, M. A.; Grosshans, P. B.; Marshall, A. G. Theoretical Mass and Energy Upper Limits for Thermal Ions in Fourier Transform Ion Cyclotron Resonance Mass Spectrometry. Int. J. Mass Spectrom. Ion Processes 1992, 120, 193-205.

35. Arkin, C. R.; Laude, D. A. Collision Induced Ion Ejection in an FTICR Trapped-Ion Cell. J. Am. Soc. Mass Spectrom. 2005, 16, 422-430.

36. Jeffries, J. B.; Barlow, S. E.; Dunn, G. H. Theory of Space-Charge Shift of Ion-Cyclotron Resonance Frequencies. Int. J. Mass Spectrom. Ion Processes 1983, 54, 169-187.

37. Francl, T. J.; Sherman, M. G.; Hunter, R. L.; Locke, M. J.; Bowers, W. D.; McIver, R. T. Experimental Determination of the Effects of Space Charge on Ion Cyclotron Resonance Frequencies. Int. J. Mass Spectrom. Ion Processes 1983, 54, 189-199.

38. Easterling, M. L.; Mize, T. H.; Amster, I. J. Routine Part-Per-Million Mass Accuracy for High-Mass Ions: Space-Charge Effects in MALDI FT-ICR. Anal. Chem. 1999, 71, 624-632.

39. Y., N.; Inoue, M. Peak Confluence Phenomenon in Fourier Transform Ion Cyclotron Resonance Mass Spectrometry. J. Mass Spectrom. Soc. Jpn. $1994,42,1-9$.

40. Marshall, A. G.; Hendrickson, C. L. Fourier Transform Ion Cyclotron Resonance Detection: Principles and Experimental Configurations. Int. J. Mass Spectrom. 2002, 215, 59-75.

41. Washburn, M. P.; Ulaszek, R. R.; Yates, J. R. Reproducibility of Quantitative Proteomic Analyses of Complex Biological Mixtures by Multidimensional Protein Identification Technology. Anal. Chem. 2003, 75, 5054-5061.

42. Gaucher, S. P.; Taylor, S. W.; Fahy, E.; Zhang, B.; Warnock, D. E.; Ghosh S. S.; Gibson, B. W. Expanded Coverage of the Human Heart Mitochondrial Proteome Using Multidimensional Liquid Chromatography Coupled with Tandem Mass Spectrometry. J. Proteome Res. 2004, 3, 495-505.

43. Breci, L.; Hattrup, E.; Keeler, M.; Letarte, J.; Johnson, R.; Haynes, P. A Comprehensive Proteomics in Yeast Using Chromatographic Fractionation, Gas Phase Fractionation, Protein Gel Electrophoresis, and Isoelectric Focusing. Proteomics 2005, 5, 2018-2028.

44. Wang, Y.; Shi, S. D. H.; Hendrickson, C. L.; Marshall, A. G. MassSelective Ion Accumulation and Fragmentation in a Linear Octopole Ion Trap External to a Fourier Transform Ion Cyclotron Resonance Mass Spectrometer. Int. J. Mass Spectrom. 2000, 198, 113-120.

45. Paul, W.; Steinwedel, H. Ein Neues Massenspektrometer Ohne Magnetfeld. Z. Naturforsch., A: Phys. Sci. 1953, 8, 448-450.

46. Campbell, J. M.; Collings, B. A.; Douglas, D. J. A New Linear Ion Trap Time-of-Flight System with Tandem Mass Spectrometry Capabilities. Rapid Commun. Mass Spectrom. 1998, 12, 1463-1474.

47. Marshall, A. G.; Wang, T. C. L.; Ricca, T. L. Tailored Excitation for Fourier Transform Ion-Cyclotron Resonance Mass Spectrometry. J. Am. Chem. Soc. 1985, 107, 7893-7897. 